\title{
PROGNOSTIC ACCURACY OF THALAMIC PROTON MAGNETIC RESONANCE SPECTROSCOPY FOLLOWING THERAPEUTIC HYPOTHERMIA IN NEONATAL ENCEPHALOPATHY
}

M. Chandrasekaran ${ }^{1}$, S. Thayyil ${ }^{1}$, A. Bainbridge ${ }^{2}$, G. Kendall ${ }^{1}$, R. Gunny ${ }^{3}$, E. Cady $^{2}$, N. Marlow ${ }^{1}$, N.J. Robertson $^{1}$

${ }^{1}$ Institute for Women's Health, University College London, ${ }^{2}$ MR Physics Department, University College London Hospital NHS Foundation Trust, ${ }^{3}$ Radiology, Great Ormond Street Hospital, London, UK

Background and aims: Deep grey matter lactate/ $\mathrm{N}$-acetylaspartate (Lac/NAA) peak area ratio on proton magnetic resonance spectroscopy $\left({ }^{1} \mathrm{H}\right.$ MRS $)$ is the best predictor of neurological outcome following neonatal encephalopathy (NE). We evaluated the prognostic accuracy following therapeutic hypothermia (TH).

Methods: We performed magnetic resonance imaging (MRI) in consecutive term babies with NE, over a 3 year period before and after introduction of TH. Death or severe neurodisability (BSID 3) at 12 months was considered as an unfavourable outcome.

Results: Of 28 babies studied, 11 babies received TH. The median (range) age at MRI was $10(5,12)$ days. Thalamic Lac/NAA $(>0.29)$ had $100 \%$ sensitivity and specificity for predicting adverse outcome in both normothermic babies and cooled infants (Table 1). The mean (SD) Lac/NAA was high in infants who had unfavorable outcome (Figure 1)

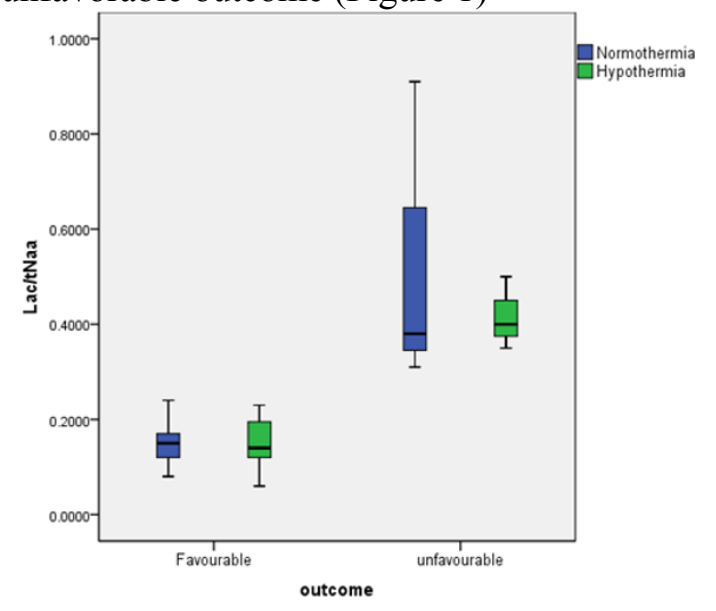

[Figure 1]

\begin{tabular}{|c|c|c|c|c|}
\hline \multirow[t]{2}{*}{ Test } & \multicolumn{2}{|l|}{ Lac/NAA } & \multicolumn{2}{|c|}{ Conventional MR } \\
\hline & Normothermia & Hypothermia & Normothermia & Hypothermia \\
\hline Sensitivity $(95 \% \mathrm{CI})$ & $100(55,100)$ & $100(57,100)$ & $100(48-100)$ & $66(24-93)$ \\
\hline Specificity $(95 \%$ CI) & $100(90,100)$ & $100(84,100)$ & $71(60-71)$ & $75(59-85)$ \\
\hline $\begin{array}{l}\text { Positive predictive } \\
\text { value }(95 \% \mathrm{CI})\end{array}$ & $100(55,100)$ & $100(57,100)$ & $42(20-42)$ & $50(18-69)$ \\
\hline $\begin{array}{l}\text { Negative predictive } \\
\text { value }(95 \% \mathrm{CI})\end{array}$ & $100(90,100)$ & $100(84,100)$ & $100(84-100)$ & $85(67-97)$ \\
\hline Area Under the Curve (SE) & $0.97(0.03)$ & $1.0(0.0)$ & $0.81(0.09)$ & $0.75(0.19)$ \\
\hline
\end{tabular}

[Table 1]

Conclusions: The prognostic accuracy of ${ }^{1} \mathrm{H}$ MRS deep gray matter Lac/NAA peak area ratios is unaltered by $\mathrm{TH}$. 